\title{
A quantitative network modeling approach to evaluate the role of cytokine combinations on CD4+ T cell differentiation, partial polarization, and plasticity
}

\author{
Mariana E. Martinez-Sanchez ${ }^{1,2}$, Leonor Huerta ${ }^{3}$, Elena R. Alvarez-Buylla ${ }^{1,2, *}$, Carlos \\ Villarreal $^{4, *}$ \\ ${ }^{1}$ Laboratorio Genética Molecular, Epigenética, Desarrollo y Evolución de Plantas, Instituto de \\ Ecología, Departamento de Ecología Funcional, Universidad Nacional Autónoma de México, Ciudad \\ de México, México. \\ ${ }^{2}$ Centro de Ciencias de la Complejidad, Universidad Nacional Autónoma de México, Ciudad de \\ México, México. \\ ${ }^{3}$ Laboratorio B108, Instituto de Investigaciones Biomédicas, Departmento de Immunología, \\ Universidad Nacional Autónoma de México, Ciudad de México, México. \\ ${ }^{4}$ Instituto de Física, Departamento de Física Cuántica y Fotónica, Universidad Nacional Autónoma \\ de México, Ciudad de México, México.
}

* Correspondence:

Carlos Villarreal_carlos@fisica.unam.mx

Elena R. Alvarez-Buylla_ eabuylla@gmail.com

Keywords: CD4+ T cells, regulatory network, ODE, heterogeneity, plasticity, microenvironment, cytokines.

\begin{abstract}
Diverse cellular polarization states with different phenotypes and functions are derived from the differentiation of activated $\mathrm{CD}^{+}{ }^{\mathrm{T}}$ naïve lymphocytes in the presence of particular cytokines. In addition, conversion of polarized cells to phenotypes different from that originally induced has been documented, highlighting the capacity of the immune response for adaptation to changing circumstances. In a recent study, we proposed a minimal Boolean regulatory network of CD4+ T differentiation that incorporates transcription factors, signaling pathways, and autocrine and exogenous cytokines. The qualitative model effectively reproduced the main polarized phenotypes of $\mathrm{CD}^{+} \mathrm{T}$ cells and several of the plasticity events reported in the literature. Yet, the amount and the expression of cytokines relative to expression of other factors influence CD4+ T cell transitions. In this paper, we have extended the Boolean network to a continuous model that allows us to assess the effect of quantitative differences in the concentrations and combinations of exogenous and endogenous cytokines, as well as diverse levels of transcription factors expression, in order to assess the role of intracellular and extracellular components in $\mathrm{CD} 4^{+} \mathrm{T}$ differentiation and plasticity. Interestingly, the model predicts either abrupt or gradual differentiation patterns between observed phenotypes depending on critical concentrations of single or multiple environmental cytokines. Plastic changes induced by environmental cytokines were observed in conditions of partial phenotype polarization in the Th1/Th2 transition. On the other hand, the Th17/iTreg transition was highly dependent on cytokine concentrations in the environment. Thus, modeling shows how the
\end{abstract}


concentration of exogenous factors, the degree of initial polarization, and cell heterogeneity, may determine the differentiation and plasticity capacity of $\mathrm{CD} 4^{+} \mathrm{T}$ cells. The model and results presented here are useful to further understand system-level mechanisms underlying observed patterns of CD $4^{+}$ T differentiation and plasticity.

\section{Introduction}

The phenotype of a cell emerges from the feedback between internal regulatory networks and microenvironmental signals. The combination, concentration, and duration of the latter can either stabilize a transcriptional profile characteristic of a particular cell lineage or promote a transition into another cell type (DuPage and Bluestone, 2016; Stockinger and Brigitta, 2010). CD4+ T cells constitute a useful model to evaluate the role of micro-environmental signals and regulatory elements on cell function. In this system, the combination and concentration of exogenous cytokines are crucial for CD4+ T cell differentiation and plasticity (DuPage and Bluestone, 2016; Stockinger and Brigitta, 2010; Eizenberg-Magar et al., 2017).

CD4+ T cells are part of the adaptive immune response. Naïve CD4+ T cells are activated in response to antigens presented by antigen presenting cells (APC) (Zhu et al., 2010). Depending on the cytokines in the microenvironment, these cells may differentiate into particular subsets. APCs are the main source of cytokines (extrinsic cytokines), but they can also be produced by other cells of the organism (Duque and Descoteaux, 2014; Sozzani et al., 2017). Exogenous cytokines bind to the membrane receptors of the cell and activate intracellular signaling pathways. These signals activate or inhibit particular transcription factors and promote the production of autocrine cytokines, creating a positive feedback that reinforces the polarization dynamics (Zhu et al., 2010). In addition, autocrine cytokines can also activate or inhibit other cells of the immune system and combinations of cytokines can have synergistic or antagonistic effects on CD4+ T cell differentiation procesess that orchestrate pathogen attack, modulation of the immune response, or immunopathology (Zhu et al., 2010).

Functional CD4+ T lymphocytes can be grouped into subsets known as Th1, Th2, Th9, Th17, Treg, $\mathrm{Tr} 1$, and Tfh. It has been documented that Th1 cells require extrinsic IL-12 and IFN $\gamma$, they express Tbet and IFN $\gamma$ (Perez et al., 1995; Hsieh et al., 1993; Szabo et al., 2000, 2003). Th2 cells require extrinsic IL-4 and are stabilized by IL-2, they express GATA3, IL-4, IL-5 and -IL13 (Cote-Sierra et al., 2004; Le Gros et al., 1990; Zheng W, 1997; Ansel et al., 2006; Swain SL Weinberg AD, 1990). Th17 cells require extrinsic TGF $\beta$ and IL-6, IL-21 or IL-23, they produce ROR $\gamma$ t, IL-21, IL-17A and IL-17F (Zhou et al., 2007; Ivanov et al., 2006; Veldhoen et al., 2006; Korn et al., 2009). Treg cells require extrinsic TGF $\beta$ and IL-2, they express Foxp3, TGF $\beta$ and in some cases IL-10 (Davidson et al., 2007; Zheng et al., 2007; Chen et al., 2003; Hori S Nomura T, 2003). Th9 cells require IL-4 and TGF $\beta$, they express IL-9 (Schmitt et al., 2014; Kaplan, 2013; Lu et al., 2012). Tr1 and Th3 cells express IL-10 and TGF $\beta$ respectively (Roncarolo et al., 2006; Awasthi et al., 2007; Gagliani et al., 2015). Tfh cells require IL-21, they express Bcl6 (Johnston et al., 2009; Nurieva et al., 2009; Yu et al., 2009; Crotty, 2014).

Furthermore, CD4+ T cells are highly heterogeneous, suggesting that cell populations go through a continuum of polarization levels after initial priming (Stockinger and Brigitta, 2010; DuPage and Bluestone,2016; Magombedze et al., 2013; Eizenberg-Magar et al., 2017). Thus, mixed cellular phenotypes may be encountered under particular cytokine concentrations and combinations, and in 
some cases, hybrid cell types such as Th1-like and Th2-like regulatory cells or Th1/Th2 hybrids (Hegazy et al., 2010; Koch et al., 2009; Wohlfert et al., 2011). Studies performed on polarized CD4+ $\mathrm{T}$ cell populations indicate that, even under controlled in vitro conditions, stimulation generates heterogeneous cell populations with variable cytokine expression profiles or intermediate cell types (Assenmacher et al., 1994; Bucy et al., 1994; Openshaw et al., 1995; Kelso et al., 1999; EizenbergMagar et al., 2017). Asymmetric cell division with segregation of signaling proteins may explain this behavior (Verbist et al., 2016).

The same cytokines responsible for the induction of naïve cells to a particular polarized state may also dictate the conversion from a different subset to this state. For example, multiple studies report the transit of Treg cells towards Th17 cells in response to the addition of exogenous IL-6 in the presence of TGF $\beta$ (Yang et al., 2008; Stockinger and Brigitta, 2010; Lee et al., 2009a). Other plastic transitions depend on the degree of polarization, as in the case of the Th17/Treg (Berod et al., 2014; Michalek et al., 2011; Gagliani et al., 2015) and the Th1/Th2 transition (Perez et al., 1995; Murphy et al., 1996; Hegazy et al., 2010). Recently polarized Th1 and Th2 cells can transdifferentiate into other subsets in response to environmental IL-4 or IL-12, but fully polarized Th1 and Th2 cells are robust to changes in the microenvironment (Murphy et al., 1996). Despite abundant experimental data, understanding of the underlying mechanisms that explain the observed differentiation and plasticity patterns of these cells must require the integrated analysis of interactions between the molecular pathways induced by microenvironmental factors, and heterogeneity in the internal capacity of cells for activation.

Complex regulatory networks give rise to stable multidimensional configurations (attractors) that correspond to expression profiles that, in this case, characterize specific CD4+ T cell subsets (Kauffman, 1969; Mendoza et al., 1999; Bornholdt, 2008; Villarreal et al., 2012; Martínez-Sosa et al., 2013; Albert and Thakar, 2014; Naldi et al., 2015; Álvarez-Buylla et al., 2016). Multidimensional Epigenetic Landscapes (EL), originally proposed as a metaphor, can be studied as a dynamical system determined by a set of coupled non-linear interactions between the network nodes that result in the emergence of a diversity of steady-state patterns; each expression pattern may be attained in a number of ways starting from different initial configurations of the expression levels of the network components. This number determines the size of the attraction basin, and consequently, the probability that a given pattern is expressed is proportional to this size. (Cortes et al., 2008; Villarreal et al., 2012; Davila-Velderrain et al., 2015). Even though the mathematical structure of the network interactions does not allow the derivation of an explicit potential function representing the EL, a probabilistic approach to the EL (not considered in this paper) is still possible (see Villarreal, 2012). On the other hand, the analyses presented here reveals the conditions required to drive the system from one attractor to another one (Haken, 1977). Indeed, the steady states (or attractors) of the dynamical system, may be visualized in as states lying at the bottom of valleys of the EL. From this point of view, we explore not only pathways that conduce to equilibrium valleys, but also alterations of the expression levels of components of the network that induce the transit between adjacent valleys.

By considering the former approach, we explore how the restrictions imposed by the regulatory network determine or affect the patterns of CD4+ T cell differentiation and plasticity (Mendoza, 2006; Naldi et al., 2010; Carbo et al., 2013; Abou-Jaoudé et al., 2014; Martinez-Sanchez et al., 2015; Eizenberg-Magar et al., 2017). With that purpose, we propose a continuous version of an already considered regulatory model (Martinez-Sanchez et al., 2015). The continuous model enables evaluations of quantitative alterations of the inputs (exogenous cytokines) and the intrinsic components (transcription factors, signaling pathways, autocrine cytokines) of the network by using a 
model of ordinary differential equations (Villarreal et al., 2012; Davila-Velderrain et al., 2015). The study contemplates a method specifically designed to study the EL repatterning under altered microenvironmental conditions (Davila-Velderrain et al.,2015; Pérez-Ruiz et al., 2015). In general, this type of systemic dynamic multi-stable models constitutes useful tools to integrate experimental data and may provide novel predictions concerning phenotypic patterns in Systems Biology. Our simulation results reproduce the main polarized phenotypes of CD4+ T cells and several of the plasticity patterns reported in the experimental literature. We determine the effect of systematic changes in the concentrations of exogenous cytokines and the internal state of the network in the differentiation and plasticity of CD4+ T cells. We focus on the Th1/Th2, and Th17/iTreg transitions, which have been thoroughly characterized given their therapeutic relevance. In addition, our study provides some novel interesting predictions that may be tested experimentally.

\section{Methods}

Similarly as the original Boolean network, its continuous variant includes nodes that correspond to transcription factors, signal transduction pathway components, cytokine receptors, SOCS proteins, as well as autocrine and exogenous cytokines [File S1]. The edges of the network correspond to the verified regulatory interactions between the nodes [Figure 1, File S2] (Martinez-Sanchez et al., 2015). In contrast with the Boolean approach, the expression level of each node may acquire continuous values within the range $(0,1)$ and the network interactions are characterized by fuzzy logic propositions. In fuzzy logic (Novak et al.,1999), the degree to which an object exhibits a property is given by a membership function (specified below) so that the truth values of variables may range between completely false (0) and completely true (1). These extreme values correspond in our system to the basal expression level (inactive) and to the maximum expression level (active) of a node, respectively. Fuzzy propositions may be built by replacing ordinary logical operations by fuzzy connectors, so that logical conjunction $A A N D B$ corresponds to the ordinary product $A * B, \operatorname{logical}$ disjunction $A O R B$ to $A+B-A * B$, and logical negation NOT $A$ to $1-A$. This methodology, discussed in Ref. (Azpeitia et al., 2014) has been used successfully for several other systems (See review in Davila-Velderrain et al., 2017, and allows to construct a parameter-free model that evidences the regulatory logics of the system and yields the observed biological behavior. The model was validated by verifying that the predicted $\mathrm{CD} 4^{+} \mathrm{T}$ subsets and plasticity transitions coincide with experimental observations [Figure 1, File S3].

The simulations performed in this work consider a reduced version of the extended T CD4+ regulatory network presented in file S1 Fig. of Ref. (Martinez-Sanchez et al., 2015). Reduction was achieved by either collapsing downstream linear signaling pathways of the regulatory network, or by using logical algebraic rules (Naldi et al., 2009; Villarreal et al., 2012). The final network consisted of 21 nodes [Fig. 1). Five nodes correspond to transcription factors (TBET, GATA3, FOXP3, RORGT, and BCL6); seven nodes correspond to signaling pathways integrating signal transducers such as STAT proteins, interleukin receptors, and autocrine cytokines (IFNg, IL2, IL4, IL10, TGFB, IL9, and IL21); nine nodes correspond to exogenous cytokines, that are produced by other cells of the immune system and that serve as inputs to the network (IFNGe, IL12e, IL2e, IL4e, IL10e, IL27e, TGFBe, IL6e and IL21e). These are marked with an "e" after the cytokine name. To study the effect of the microenvironment we focused on nine biologically relevant environments (Zhu et al., 2010): pro-Th0, pro-Th1, pro-Th2, pro-Th17, pro-Th9, pro-Tfh, pro-iTreg, pro-Tr1, and pro-Th3 [Table 1]. The regulatory cytokine IL-10 deserves special consideration, since it uses STAT3, similarly as the 
inflammatory cytokines IL-6 and IL-2. Thus, we assume that IL-10 signaling is mediated by an independent pathway than IL-6/IL-21, even though they share STAT3 as a messenger molecule (Moore et al., 2001). While IL-27 has been linked to multiple functions, we consider that its main role in the model is a regulatory cytokine (Pot et al., 2009; Murugaiyanet al., 2009; Awasthi et al., 2007). The model ignores weak interactions, chemokines, and epigenetic regulation that are also relevant and should be included in future modeling efforts.

\begin{tabular}{|l|l|}
\hline Environment & Active input nodes \\
\hline pro-Th0 & None \\
\hline pro-Th1 & IFNge, IL12e \\
\hline pro-Th2 & IL2e, IL4e \\
\hline pro-Th17 & IL21e, TGFBe \\
\hline proTh9 & IL4e, TGFBe \\
\hline proTfh, & IL21e \\
\hline pro-iTreg & IL2e, TGFBe \\
\hline pro-Tr1 & IL10e, IL27e \\
\hline pro-Th3 & TGFBe \\
\hline
\end{tabular}

Table 1: Exogenous cytokines in different environments included in the CD4+ T cell regulatory network. Active nodes refer to the same exogenous cytokines, whose concentrations were modified during the simulation, adopting values between 0 and 1 .

If we now assume that $q_{i}(t)$ represents the expression level of node $i$ at a given time, its rate of change determined by the regulatory network interactions may be described by

$$
\frac{\mathrm{dq}_{i}(t)}{\mathrm{dt}}=\frac{1}{1+\exp \left[-b\left(w_{i}-w_{i}^{\mathrm{thr}}\right)\right.}-\alpha_{i} q_{i}(t)
$$

Here, the first term on the right hand side represents a (logistic) membership function discussed above. It depends on the fuzzy logic proposition $\mathrm{w}_{\mathrm{i}}$, with a threshold value $\mathrm{w}_{\mathrm{i}}{ }^{\text {thr }}$ that renders the proposition as true. The parameter $b$ is a rate indicating the rapidity of the transition from false to true. Finally, $\alpha_{\mathrm{i}}$ is a decay rate, so that if the membership degree is null, the node expression level decays exponentially at this rate. In this paper we suppose that $\alpha_{i}=1, w_{i}{ }^{\text {thr }}=1 / 2$, and $b=25$. The resulting attractors of the dynamical system are presented in [File S4].

The continuous model enables evaluations of quantitative alterations of the inputs (exogenous cytokines) and the intrinsic components (transcription factors, signaling pathways, autocrine cytokines) of the network. To investigate the polarization process we assume an initial state that corresponds to a $\mathrm{CD}^{+}{ }^{+} \mathrm{T}$ cell activated under non-polarizing cytokine conditions (Th0) where we suppose that nodes representing transcription factors, signaling pathways or autocrine cytokines are initially inactive. In order to model plastic transitions, we consider a cell in an initial polarization state determined by different expression levels of the characteristic transcription factor and cytokines [File S3] in the range $0 \geq q_{i}$ int $\geq 1$. In both kinds of experiments, we represent the effect of the microenvironment using a selected set of exogenous cytokines [Table 1] that are active with relative concentrations lying in the range $0 \geq \mathrm{q}_{\mathrm{i}}{ }^{\text {ext }} \geq 1$. For each initial condition and relative concentration of 
the microenvironment, we simulate the system to obtain the final steady state and measure the Euclidean distance between the initial state and the final steady state.

We consider that a steady state of the system corresponds to a CD4 ${ }^{+} \mathrm{T}$ cell subset if its characteristic transcription factor and cytokines [File S5] are actively expressed. Given the continuous range of expression levels in the system, we introduce a criterium to define node expression. We consider that a node is actively expressed if $q_{i} \geq 0.75$, unexpressed if $q^{i} \leq 0.25$, while intermediate values correspond to a transition zone, with no definite expression. We consider that an attractor corresponds to a given subset if the characteristic cytokines and transcription factors are active. On these terms, a cell was classified as Th0 if it expressed low levels $\left(\mathrm{q}_{\mathrm{i}}<0.25\right)$ of transcription factors [File S5].

We observe that the polarization transitions between steady states of the system display two alternative behaviors as a function of exogenous cytokine concentrations. In the first case, a sudden (discontinuous) transition appears at a critical concentration of polarization inducing cytokines. In the second case, the transition is gradual (continuous), involving a transition region with intermediate expression levels of endogenous components, which could be interpreted in terms of phenotype heterogeneity. The code for all the simulation experiments performed in this work is available in [File S6].

\section{Results}

\section{Network construction}

A previous study reported a data-based Boolean regulatory network for CD4+ T cell differentiation and plasticity [File S1 and S2]. While the Boolean model correctly reproduces CD4+ T cell fate attainment, it is unable to determine how the different expression levels of the components of the network (transcription factors, signaling pathways, autocrine and exogenous cytokines) affect CD4+ T cell differentiation and plasticity. To address this, we converted the equations to a continuous dynamical model [File S4] (Mendoza,2006; Villarreal et al., 2012), and used the EL reshaping methodology (Davila-Velderrain et al., 2015) to address which combinations and quantitative alterations of cytokine and transcription factors expression levels are sufficient for CD4+ T differentiation and plasticity.

The stable states or attractors to which the regulatory network converges are interpreted as the characteristic expression profiles of particular CD4+ T cell subsets or types (Mendoza et al., 1999; Naldi et al., 2015; Albert and Thakar, 2014; Bornholdt, 2008; Azpeitia et al., 2011; ÁlvarezBuylla et al., 2016). Given the continuous nature of the regulatory network model presented here, it is impossible to determine all the possible steady states. It was verified that the steady states of the discrete model could be recovered in the continuous model, and they were classified into CD4+ T cell subsets. It was considered that a steady state corresponded to a subset if it expressed a high concentration (qi> 0.75) of the characteristic cytokine and transcription factor associated with the subset, which in this work corresponded to Th0, Th1, Th2, Th17, Treg, Tfh, Th9, Tr1, and Th3. We also recovered steady states that correspond to GATA3+IL4-, Th1-like and Th2-like regulatory cells, which had been previously reported (Martinez-Sanchez et al.,2015; DuPage and Bluestone, 2016). 
Steady states that had intermediate values were considered to be in a transition zone (t.z.) of phenotypic coexistence.
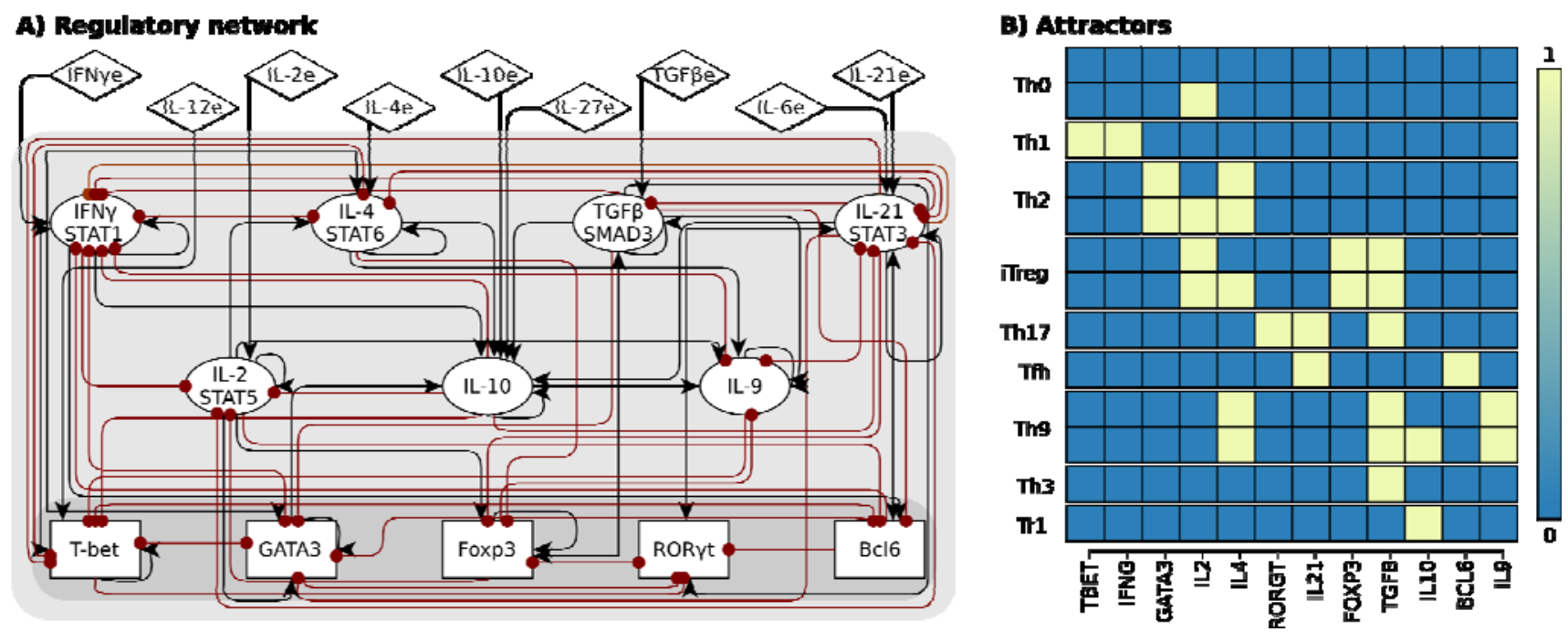

Figure 1. CD4+ T cell transcriptional-signaling regulatory network. The regulatory network was constructed using available experimental data. (A)The network includes transcription factors (rectangles), autocrine cytokines and their signaling pathways (ellipses) and exogenous cytokines (diamonds). Interactions leading to activation are represented by black arrows, while those leading to inhibition with red dots. (B) Sample attractors of the system.

\section{Effect of the concentration of the extrinsic cytokines in the differentiation of CD4 + T cells}

To evaluate how the concentrations of the exogenous cytokines in the environment shapes $\mathrm{CD}^{+} \mathrm{T}$ cell differentiation, we studied the activation process of a Th0 cell as a function of increasing concentrations of the exogenous cytokines and determined the final steady states [Figure 2]. The exogenous cytokines IL12e, IFNGe, IL4e, IL6e, IL21e, TGFBe, and IL10e induce the differentiation from a Th0 initial steady state towards Th1, Th2, Tfh, Th3, and Tr1 subsets respectively (Zhu et al., 2010). On the other hand, Th17, Th9, and iTreg subsets were not induced by a single exogenous cytokine in the micro-environment. These observations are consistent with the requirement of TGFBe in combination with IL6e/IL21e, IL4e or IL2e to induce these subsets. (Zhu et al., 2010).

The critical concentration required to induce a transition varied depending on the particular exogenous cytokine. IL12e, IL6e, and IL21e required relatively small concentrations (0.2) to induce the differentiation from Th0 to Th1 and Tfh respectively, while IL4e required a higher concentration (0.36) to induce the differentiation from $\mathrm{Th} 0$ to $\mathrm{Th} 2$. On the other hand, IL2e and IL27e alone were not able to induce transitions. We observed that IL2e induced the expression of high levels of IL2; however, we labeled the resulting cells as Th0, as IL-2 production by itself is not associated with a particular polarization subset.

It is also interesting to note that transitions among subsets have different patterns of sensitivity to exogenous cytokine concentrations. Most of the transitions from Th0 to other subsets were discontinuous; once a threshold concentration was achieved, the cell changed its expression 
pattern to a different one in an abrupt manner. An exception was observed when IL10 was used as an inducer. This cytokine caused a gradual transition from Th0 to Tr1; in this case, a continuous range of steady states is attained in the transition zone between both subsets. These results show that, for most of single cytokines, $\mathrm{CD} 4^{+} \mathrm{T}$ cells would initiate differentiation once the threshold concentration has been reached, whereas they may display a range of sensivities to the concentration of other cytokines to induce differentiation into alternative phenotypes.
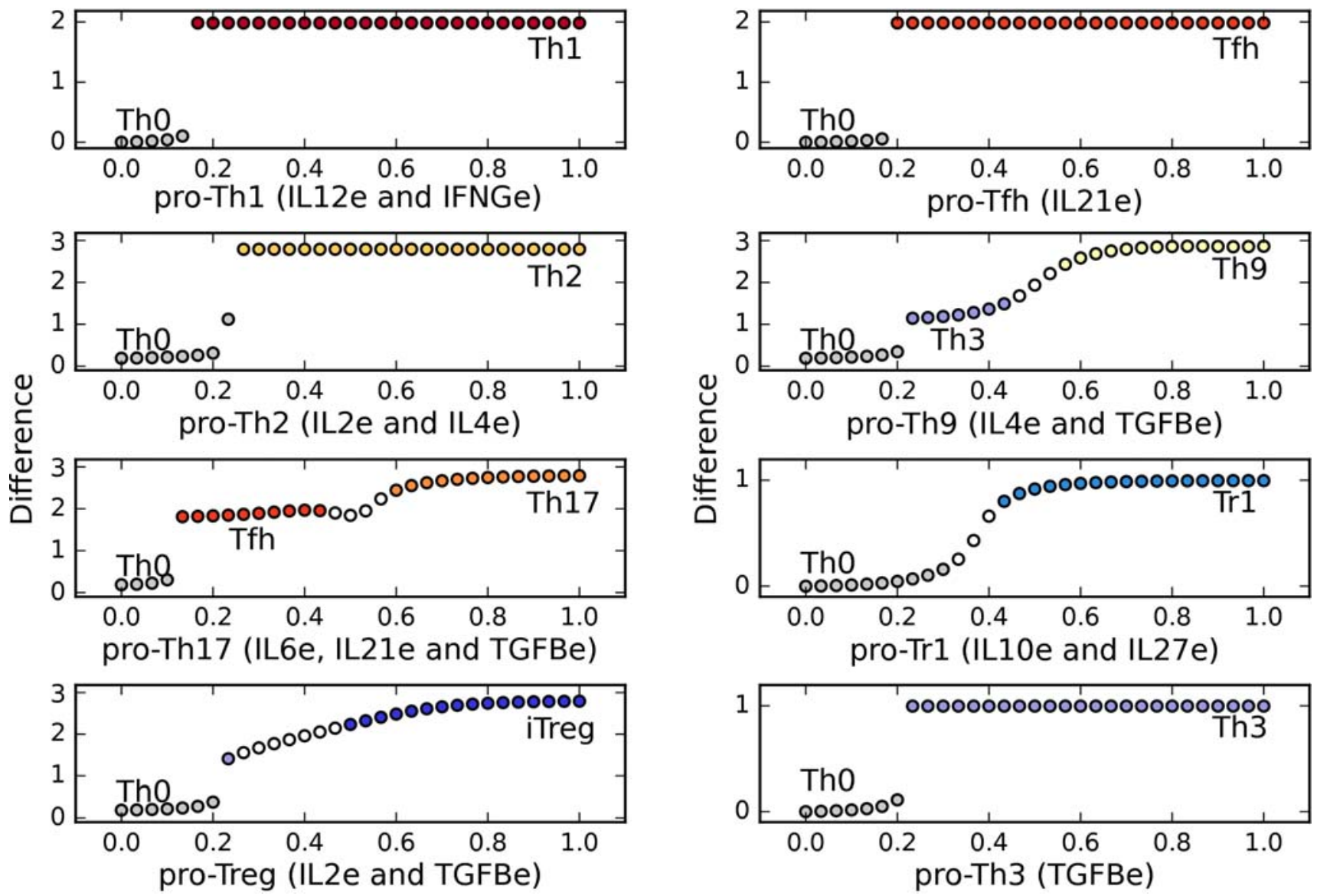

Figure 2. CD4+ T cell fate as a function of the concentration of single exogenous cytokines: IL12, IFNG, IL2, IL4, IL6, IL21, TGFB, IL10, IL27. From an initial state TH0, a CD4+ T cell may acquire diverse phenotypes on an abrupt or gradual transition, depending on critical concentrations of environmental cytokines. We observe that the presence of either IL12 or IFNg is sufficient for Th1 polarization, as well as IL4, is sufficient for TH2 polarization. On the other hand, IL2 alone does not lead to an effector phenotype. Similarly, the presence of either IL6 or IL21 alone is sufficient for Tfh induction, as is the case of TGFB and IL10, leading to Th3 and Tr1, respectively. IL27 alone does not lead to any fate transition in this model.

$\mathrm{CD}^{+}{ }^{+} \mathrm{T}$ subsets such as Th9, Th17, and iTreg require combinations of cytokines to differentiate from naïve cells. In our model, we simulated the activation of a Th0 cell in the presence of different combinations and concentrations of the exogenous cytokines associated with the microenvironment [Table 1, Figure 3]. In the case of requiring more than one exogenous cytokine, all the implicated nodes were set to the same value. Using this methodology, we were able to induce the differentiation from a Th0 steady state towards Th1, Th2, Th17, Th9, Tfh, iTreg, Th3, and Tr1 subsets by cytokine combinations that are in agreement with experimental data (Zhu et al., 2010, Crotty2014, DuPage2016c). 
The concentration required to induce transitions when using multiple cytokines varied depending on the $\mathrm{CD}^{+}{ }^{+} \mathrm{T}$ cell type, but it was always lower [Figure 2] than if only a single exogenous cytokine was added. This result suggests that the underlying $\mathrm{CD}^{+} \mathrm{T}$ cell differentiation regulatory network mediates a synergistic effect of cytokines on $\mathrm{CD} 4^{+} \mathrm{T}$ cell differentiation. For example, while a concentration of IL $4 \mathrm{e}=0.36$ was necessary to induce the polarization towards Th2, a concentration of IL $2 \mathrm{e}$ and IL4e $=0.26$ was sufficient to induce the same transition. Similarly, while a concentration of IL10e $=0.6$ was necessary to induce the polarization towards $\operatorname{Tr} 1$, a concentration of IL10e and IL27e $=0.43$ produced the same transition. Furthermore, autocrine IL10 achieved its maximum value with a lower concentration of exogenous cytokines when IL10e and IL27e act synergistically.

The transitions in pro-Th1, pro-Th2, and pro-Tfh microenvironments were abrupt, while the transition in a pro-Tr1 environment was gradual. In a pro-Th17, pro-Th9, and pro-iTreg microenvironments, all including TGFße, there was a small abrupt change followed by a gradual change in the expression values of the steady state. Also, in pro-Th17 and pro-Th9 we found an intermediate step before the final polarized state. In the pro-Th17 case, increasing cytokine levels induced an initial abrupt change towards a plateau zone corresponding to Tfh, followed by a transition to the Th17 steady state. A similar behavior was observed in the pro-Th9 microenvironment with a precursor TGF $\beta^{+}$(Th3) subset, followed by a final Th9 steady state. It is worth noting that TGF $\beta$ has a key role in the induction of the three types of $\mathrm{CD}^{+} \mathrm{T}$ cell types discussed here trough complex relationship with the effect of other exogenous cytokines (Eizenberg-Magar et al., 2017). These results illustrates the capability of the minimal model to contribute to the understanding of how the context determine the cellular response to TGF $\beta$ in the immune system. 
Quantitative network model of CD4+ T cells
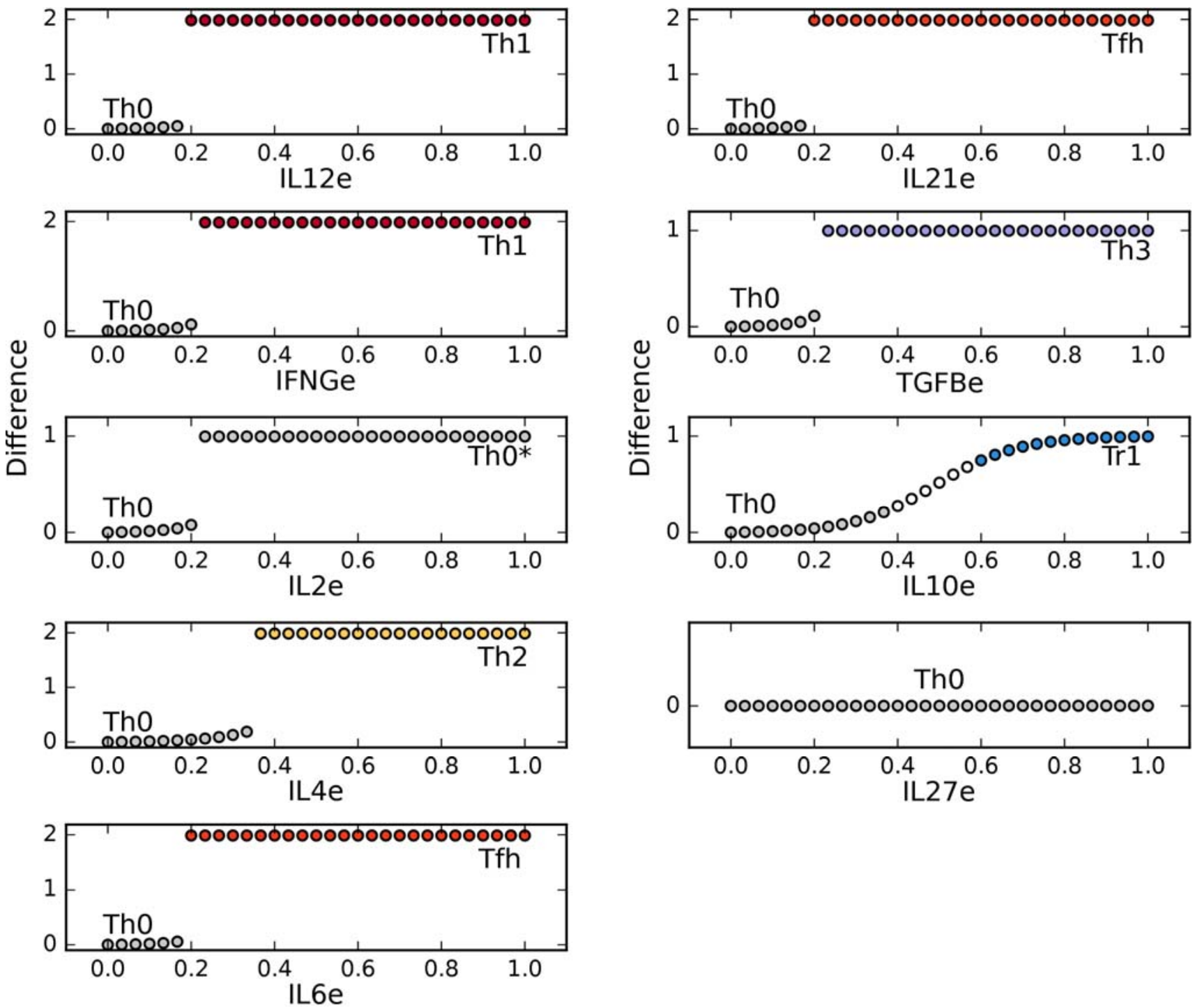

Figure 3. T-CD4 cell fate as a function of exogenous cytokine concentrations defining diverse phenotype-associated environments. From the THO initial state, a T CD4 cell evolves to different phenotypes, depending on critical concentrations of environmental cytokines as shown in Table 1: Th1 (IFNG and IL12), Th2 (IL4, Il2), Th17 (Il21, TGFB), Treg (IL2, TGFB), Tfh (IL21), Th9 (IL4, TGFB), Tr1 (IL10, IL27), Th3 (TGFB).. The transition may be abrupt or gradual and, interestingly, may involve an intermediate state, as in the cases Th0 -> Tfh $\rightarrow$ Th17 (C), and Th0 -> Th3 $>$ Th9 $(\mathrm{F})$.

Thus, the continuous model describes the cytokine concentration dependence of the differentiation of $\mathrm{T}$ cell subsets, illustrating abrupt or gradual changes, the effect of cytokine combinations and, notably, the induction of different subsets under the action of different concentrations of the same cytokine combinations. 


\section{Effects of the concentration of the exogenous and endogenous cytokines and transcription factors in on the plasticity of $\mathrm{CD4}^{+} \mathrm{T}$ cells}

Once differentiated, $\mathrm{CD}^{+} \mathrm{T}$ cells may undergo plastic transitions to other subsets under specific conditions. As discussed above, in order to model this kind of transitions we studied phenotypic alterations of cells stated in a defined polarization state, as a function of different concentration levels of exogenous cytokines able to induce an alternative phenotype.

We first focus on the transition between Th1 and Th2 [Figure 4], which has been experimentally observed, particularly when these cells have recently differentiated (Perez et al., 1995; Panzer et al., 2012). To study this process we considered the response of already differentiated Th1 and Th2 states, to the presence of variable concentrations of the of the subset-defining cytokine in combination with the opposing cytokine (IFNGe for Th2, and IL4e, for Th1), and determined the final steady state. When the initial configuration of the system corresponded to a highly polarized Th1 (TBET and IFNG = 1) or Th2 (GATA3 and IL4 = 1) states, for every combination of (exogenous) IL4e and IFNGe concentrations, the system remained in its original state even under high concentrations of these cytokines, respectively, indicating that highly polarized Th1 or Th2 cells are not plastic. However, by considering initial lower concentrations of Th1 and Th2 transcription factors and cytokines, consistent with partial phenotype polarization, plastic transitions ensue. CD4 ${ }^{+}$ $T$ cells require the production of high levels of autocrine IFNG and expression of TBET to maintain a Th1 phenotype. If the expression levels decrease, especially in the case of autocrine IFNG, it will transit into a Th2 cell. At the same time, the cells require the production of high levels of autocrine IL4 and expression of GATA3 to maintain a Th2 phenotype. If the initial expression levels decrease it will transit into a Th1 cell. At high expression levels of initial GATA3 and low initial IL4, there exists a transition zone where the cell displays mixed characteristics. These results show that plasticity between the Th1 and Th2 subsets depends not only on the cytokines present on in the microenvironment but also on the intracellular state. 


\section{A Initial state: Th0}
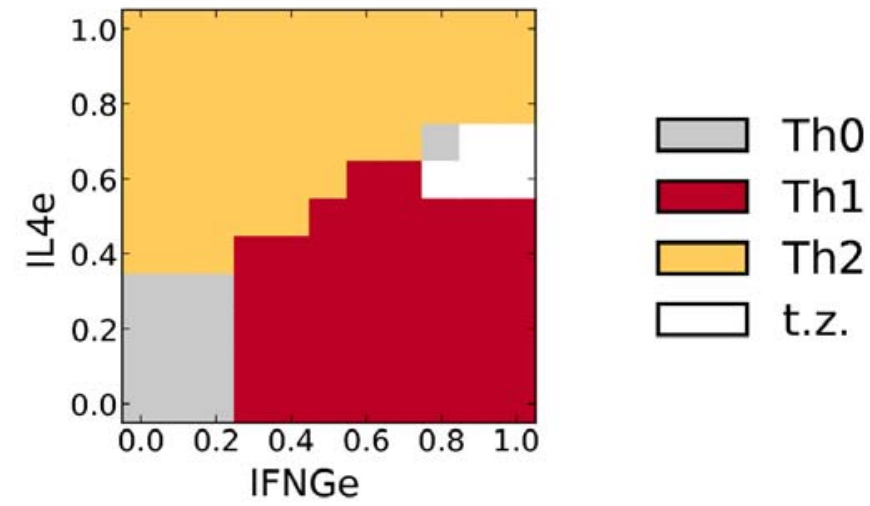

B Initial state: Th1

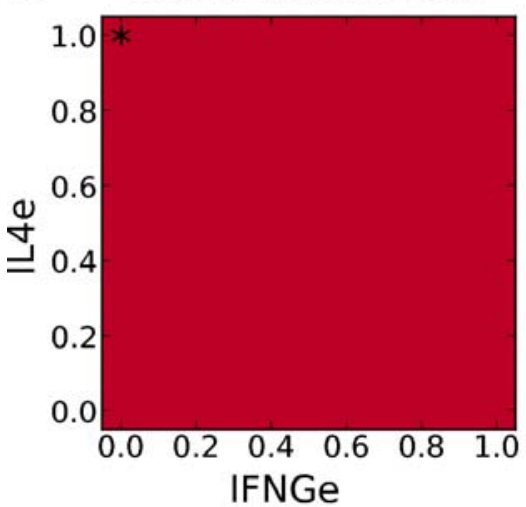

D Environment: IL4e+

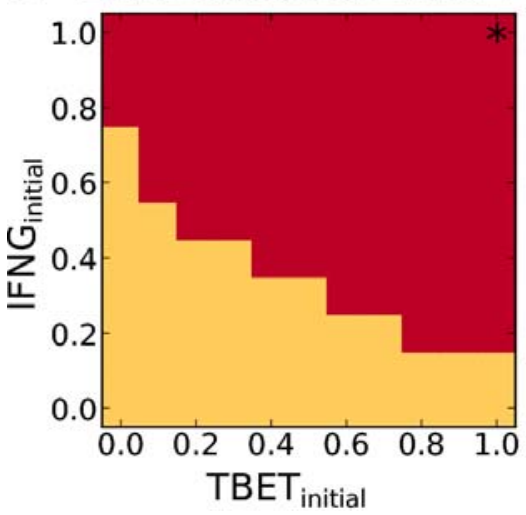

C Initial state: Th2

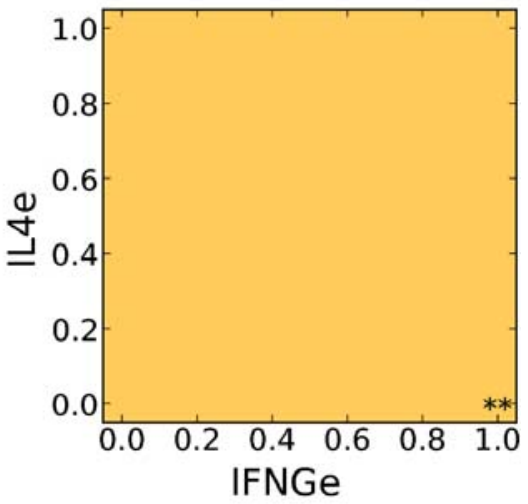

E Environment: IFNGe+

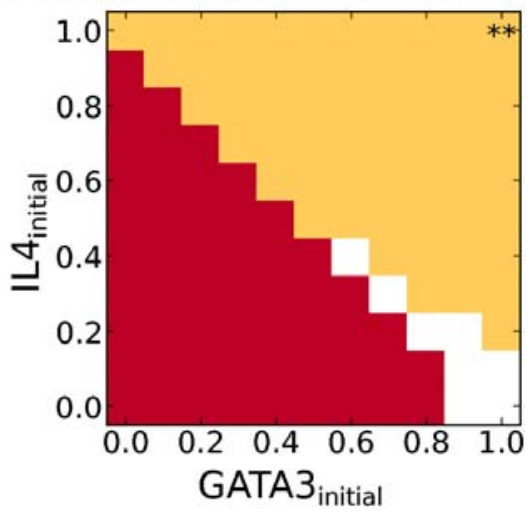

Figure 4. Phenotype space diagrams for Th1 and Th2 polarization and plasticity as a function of the relative concentration of environmental IFNg and IL4, and expression of transcription factors. (A) Diagram for cell differentiation assuming an initial Th0 state. As the external concentration of IFNGe increases, the system develops an abrupt transition from Th0 to Th1. Similarly, an increase in external IL4e drives an abrupt transition from TH0 to Th2. For moderate concentrations of IFNGe and IL4e $(<0.8)$, we observe two wide zones of Th1 or Th2 prevalence with a sharp boundary, meaning that small variation of cytokines at this zones may change cell polarization. A transition zone with no defined polarization appears at higher concentrations of these cytokines (white and gray areas). (B and C) Plasticity diagrams assuming full Th1 (B) or Th2 (C) polarized states (i.e., induced by INFg=1 and IL-4=1 in diagram A, respectively). No phenotypic transitions are observed under variable concentrations of environmental IL4e and autocrine IFNGe. (D) Plasticity diagram of Th1 cells assuming an environmental concentration of IL $4 \mathrm{e}=1$. Cells require the production of initial high levels of autocrine IFNG and expression of TBET to maintain a Th1 phenotype. If the initial expression levels decrease, especially in the case of autocrine IFNG, it will transit into a Th2 cell. (E) Plasticity diagram 
of Th2 cells assuming an environmental concentration of IFNGe $=1$. The cell requires the production of high levels of autocrine IL4 and expression of GATA3 to maintain a Th2 phenotype. If the initial expression levels decrease it will transit into a Th1 cell. At high expression levels of initial GATA3 and low initial IL4, there exists a transition zone where the cell cannot be classified.

The transition between Th17 and iTreg, has been extensively investigated experimentally (Lee et al.,2009b; Littman and Rudensky, 2010; Wei et al., 2008; Xu et al., 2007; Noack and Miossec, 2014; Leeet al., 2009a; Kleinewietfeld and Hafler, 2013) [Figure 5] since this plasticity case is particularly important for some pathological conditions, such as chronic inflammation. To study this process we considered fully differentiated Th17 (RORGT and IL21=1) and iTreg (FOXP3 and TGFB = 1) under the presence of different concentrations of the exogenous cytokines IL2e, IL21e, and TGFBe. In the case of Th17 cells, they remained in a Th17 phenotype at a high concentration of TGFBe, while they switched towards Tfh for lower concentrations of TGFBe $(<0.6)$. Some experiments have reported that induction of Th17 require exogenous TGFB (Veldhoen et al., 2006), but it is uncertain if the transition towards Tfh associated to low TGFB levels will occur in all cases. On the other hand, iTreg cells remain stable provided that high concentrations of IL2e are present, while they transit towards Th17, Tfh or Th3 when concentrations of IL2e are low $(<0.65)$. This shows that the plastic transitions between subsets are not symmetrical, and depend on the previous polarization state of the cell.
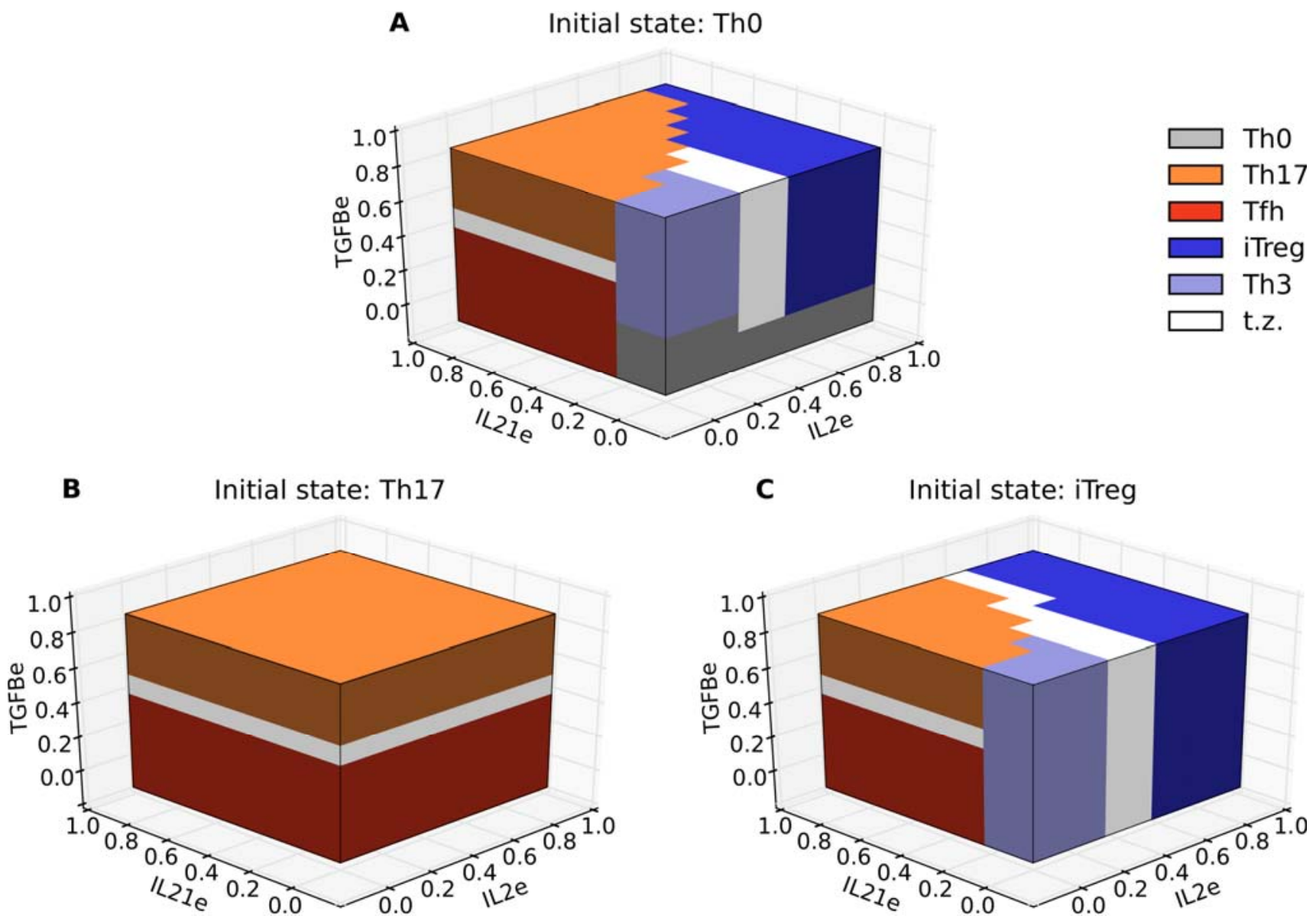

Figure 5. Three-dimensional phenotype space diagrams for Th17 and iTreg polarization and plasticity as a function of the relative concentrations of $I L 2$, IL21, and TGFB in the microenvironment. In the differentiation diagram (A) we observe alternative phenotypic regions defined by relative concentrations of environmental cytokines. 
The regions may be either separated by a sharp boundary or by a more gradual transition zone (labeled in white). The plasticity diagram (B) indicates a polarized behavior for Th17 versus Tfh phenotype determined by a high or low concentration of external TGFB. A richer behavior ensues when the initial state is Treg, as shown in the plasticity diagram (C). We observe a similar structure as that depicted in (A), except that the Th0 zone is absent.

\section{Discussion}

Our simulations show different profiles of differentiation patterns of CD4+ T cells that depend on the concentration and combinations of exogenous cytokines, highlighting the importance of synergy and competing interactions at the signaling network level. We also showed that plasticity between the Th1/Th2 and iTreg/Th17 subsets can be obtained by varying the concentration of microenvironmental cytokines and the expression level of transcription factors and autocrine cytokines derived from the internal state of the cell.

The model predicts both abrupt and gradual transitions between cell types. In abrupt transitions, there is a sudden change in the final steady state or cell type, once the concentration of exogenous cytokines exceeds a threshold value. This behavior suggests that the transition between stable cell phenotypes is energetically favorable once the threshold value has been achieved. In this process, exogenous cytokines provide the initial stimulus to promote the expression of both transcription factors and autocrine cytokines in positive feedback loops that increase the stability of attractors to quickly attain polarization.

In contrast, in gradual transitions, steady states that express intermediate levels of transcription factors and autocrine cytokines appear. In these steady states, a clear-cut threshold between the two expression patterns is not observed, so they cannot be easily classified into one subset or another, signaling the manifestation of partially polarized states. The heterogeneity of CD4+ T cells has been well-documented (DuPage and Bluestone, 2016; Stockingerand Brigitta, 2010; Eizenberg-Magar et al., 2017), and could be the result of regulatory circuits capable of generating a range of cells that express intermediate levels of specific molecules and that can stably coexist or change from one another under certain conditions. It is important to notice that every gradual transition involves regulatory circuits with central nodes which display feedback interactions. This feedback renders stability to the initial polarization state so that its intrinsic cytokine production and transcription factor expression should gradually decrease under changing microenvironmental conditions. We observed this behavior especially in response to changes in the concentration of IL-10 and TGF $\beta$. IL-10 is a regulatory cytokine produced by multiple CD4+ T subsets (Gagliani et al., 2015; Howes et al., 2014). TGF $\beta$ may display both regulatory and inflammatory effects and it is implied in the differentiation of multiple subsets like Th17, iTreg, and Th9 (Veldhoen et al., 2006; Davidson et al., 2007; Chen et al., 2003; Kaplan, 2013). It is conceivable that gradual transitions and generation of intermediate polarization states reflect the intricate regulatory signaling effects of TGF $\beta$ of IL-21, which may participate in a biological mechanism of tuning their effect in the immune response (Grossman \& Paul, 2015).

The model also captures some cases where there is an abrupt transition followed by a gradual transition in polarization processes. Such is the case of the Th0-Tfh-Th17, the Th0Th3(TGFB+)-Th9 and the Th0-iTreg transitions. Interestingly, in all these cases TGF $\beta$ is present in the micro-environment. This indicates that the concentration of TGF $\beta$ may modulate the immune response in complex ways, putting forward a system-level explanation of experimental results. It is known that TGF $\beta$ regulates Th17 cells in a differential way depending on the concentration and 
cytokines in the environment (Yang et al., 2008). Furthermore, consistent with our simulations, it is known that the TGF $\beta$ signaling pathway is highly modulated (Attisano and Wrana, 2002; Travis and Sheppard, 2014). Our model also predicts that that TGF $\beta$ may induce distinct subsets at different concentrations, in particular, Tfh, Th9, iTreg, and Th3. Studying this effect would require polarization experiments with careful control of extracellular TGF $\beta$ concentration.

The model highlights the cooperation between exogenous cytokines during differentiation. Th17, iTreg, and Th9 subsets require TGF $\beta$ in combination with IL-6/IL-21, IL-2 and IL-4 to differentiate, respectively, in agreement with experimental data (Veldhoen et al., 2006; Davidson et al., 2007; Chen et al., 2003; Kaplan, 2013). In other cases, the effect of a single cytokine is sufficient to induce polarization, but the synergy with other cytokines lowers the threshold concentration necessary to induce polarization. In this way, the model allows us to quantitatively study and predict synergistic relations among cytokines in CD4+ T cell differentiation.

As mentioned above, we also use the model to study the effect of opposing cytokines in the differentiation and plasticity of Th1/Th2 and Th17/iTreg subsets. The Th1 and Th2 cells are highly stable, and the transition between them is hard to achieve experimentally (Perez et al., 1995; Murphy et al., 1996; Hegazy et al., 2010). We found that, once they have achieved a stable state, Th1 and Th2 are robust to changes in their microenvironment. However, partially polarized cells can transit to the other cell types when they are subject to an opposing cytokine (IL-4 in the case of Th1 or IFN $\gamma$ in the case of Th2). These results agree with the observation that recently polarized Th1 and Th2 cells are plastic, but fully polarized are not (Murphy et al., 1996). This behavior seems consistent with a particularly robust interaction circuit, defined by four regulatory switching modules between mutually inhibitory nodes with negative feedback, each node defining an alternative regulatory route.

On the other hand, iTreg and Th17 cells are less stable, and there are multiple reports of single cell transitions caused by changes in the environment, especially from iTreg to Th17 cells (Lee et al.,2009b; Littman and Rudensky, 2010; Wei et al., 2008; Xu et al., 2007; Noack and Miossec, 2014; Lee et al., 2009a; Kleinewietfeld and Hafler, 2013). Our model shows that the Th17 phenotype is stable in the presence of TGF $\beta$, whereas it transits towards Tfh cells in the absence of exogenous TGF $\beta$. While this transition has been reported (Crotty, 2014), we cannot test its stability in the long term, as the model lacks many of the niche signals that stabilize Tfh cells Icite\{Crotty2014, DuPage2016c . Modeling also shows that maintenance of the iTreg phenotype requires high concentrations of IL-2, which is in accordance with experimental observations (Veldhoen et al., 2006; Davidson et al., 2007; Chen et al., 2003), although they transit towards Th17 in the presence of high concentrations of IL-21 and low concentrations of IL-2. Thus, the model provides a simulation of the spontaneous transition of iTreg into Th17 in the presence of IL-21 or the closely similar IL-6 (here considered as equivalents) (Xu et al., 2007) at low concentrations of IL-2. The plasticity of this transition is not symmetrical, as changes in the microenvironment are not enough for Th17 to transit towards iTreg. For such transition, it is also necessary to alter the internal state of the cell, changing the expression levels of key transcription factors, as has been shown in experimental studies (Berod et al., 2014; Michalek et al., 2011; Gagliani et al., 2015). These results seem to imply that the basin of attraction of iTreg is shallower than that of Th17. This could be the result of the different regulatory circuits implied in the differentiation of each cell type, since while both depend on TGF $\beta$, iTreg both requires and inhibits the production of IL-2 (Pandiyan et al., 2007; Fontenot JD Gavin MA, 2003), restricting the stability of this cells.

The model and simulations presented here are able to describe cell type transitions and do not rely upon specific parameter estimates. However, the exact transition points may change 
depending on the precise concentrations and parameters of the biological system (Eizenberg-Magar et al., 2017). In our model, the value 0 corresponds to a basal level of expression, not to the absolute absence of the transcription factor or cytokine. Given the relative nature of the quantitative variations introduced in the model, we should be cautious in providing precise quantitative predictions concerning the sensitivity of the different subsets under real experimental conditions.

The model and simulations presented here predict two main kinds of transitions between attractors: abrupt transitions, characterized by well-defined transcriptional profiles, and gradual transitions, with a zone associated to heterogeneous populations. We propose that the second kind is associated with feedback regulatory interactions as well as switching modules involved in the dynamics of the system. A careful analysis of this kind of regulatory circuits will shed light on the specific mechanisms defining transcriptional programs that lead to cell heterogeneity. This is especially important in the case of TGF $\beta$, which has a crucial role in the regulatory network. Understanding the interactions underlying the dynamical behavior may help elucidate the regulatory role of this important molecule in the immune response.

Theoretical models like the one presented here provide an ideal tool to integrate recent advances in experimental knowledge and provide a system-level mechanistic explanation for observed behaviors in experiments, and also to provide informed predictions for future experiments. Hence, the feedback between experimental and theoretical research is necessary to understand the rich behavior of CD4+ T cells and the immunological system.

\section{Conflict of Interest}

The authors declare that the research was conducted in the absence of any commercial or financial relationships that could be construed as a potential conflict of interest.

\section{Author Contributions}

ERAB and CV conceived, planned and coordinated the study. CV and MEMS established the continuous model and performed simulations and calculations. All of the authors participated in the interpretation, result analysis, and wrote the paper.

\section{Funding}

ERAB and MEMS received funding from CONACYT: 240180, 180380, 2015-01-687 and UNAMDGAPA-PAPIIT: IN211516, IN208517, IN205517, IN204217. CV received funding from CONACYT: 180380. LH received funding from CONACYT: CB2014/238931 and UNAM-PAPIIT IN211716.

\section{Acknowledgments}

We acknowledge Diana Romos for her support with logistical tasks. We thank Jose Davila-Velderrain and Juan Arias del Angel for providing code for this project. 


\section{References}

Abou-Jaoude, W., Monteiro, P. T., Naldi, A., Grandclaudon, M., Soumelis, V., Chaouiya, C., et al. (2014). Model checking to assess T-helper cell plasticity. Frontiers in bioengineering and biotechnology 2, 86. doi:10.3389/fbioe.2014.00086

Albert, R. and Thakar, J. (2014). Boolean modeling: A logic-based dynamic approach for understanding signaling and regulatory networks and for making useful predictions. Wiley Interdisciplinary Reviews: Systems Biology and Medicine 6, 353-369. doi:10.1002/wsbm.1273

Alvarez-Buylla, E. R., Davila-Velderrain, J., and Martinez-Garcia, J. C. (2016). Systems Biology Approaches to Development beyond Bioinformatics: Nonlinear Mechanistic Models Using Plant Systems. BioScience 66, 371-383. doi:10.1093/biosci/biw027

Ansel, K. M., Djuretic, I., Tanasa, B., and Rao, A. (2006). Regulation of TH2 Differentiation and il4 locus accessibility. Annual Review of Immunology 24, 607-656.

doi:10.1146/annurev.immunol.23.021704. 115821

Assenmacher, M., Schmitz, J., and Radbruch, A. (1994). Flow cytometric determination of cytokines in activated murine T helper lymphocytes: Expression of interleukin-10 in interferon and in interleukin 4 expressing cells. European Journal of Immunology 24, 1097-1101.

doi:10.1002/eji.1830240513

Attisano, L. and Wrana, J. L. (2002). Signal transduction by the TGF-beta superfamily. Science (New York, N.Y.) 296, 1646-1647. doi:10.1126/science.1071809

Awasthi, A., Carrier, Y., Peron, J. P. S., Bettelli, E., Kamanaka, M., Flavell, R. A., et al. (2007). A dominant function for interleukin 27 in generating interleukin 10-producing anti-inflammatory $\mathrm{T}$ cells. Nature immunology 8, 1380-9. doi:10.1038/ni1541

Azpeitia, E., Benitez, M., Padilla-Longoria, P., Espinosa-Soto, C., and Alvarez-Buylla, E. R. (2011). Dynamic network-based epistasis analysis: boolean examples. Frontiers in plant science 2, 92. doi:10. 3389/fpls.2011.00092

Azpeitia, E., Davila-Velderrain J.,Villarreal, C., and Alvarez-Buylla, E.R. (2014).Methods in Molecular Biology, Ed. by JL Riechmann and F. Wellmer, Springer, NY

Berod, L., Friedrich, C., Nandan, A., Freitag, J., Hagemann, S., Harmrolfs, K., et al. (2014). De novo fatty acid synthesis controls the fate between regulatory $\mathrm{T}$ and $\mathrm{T}$ helper 17 cells. Nature Medicine 20, 1327-1333. doi:10.1038/nm.3704

Bornholdt, S. (2008). Boolean network models of cellular regulation: prospects and limitations. Journal of the Royal Society, Interface / the Royal Society 5 Suppl 1, S85-S94.

doi:10.1098/rsif.2008.0132.focus

Bucy, R. P., Panoskaltsis-Mortari, A., Huang, G., Li, J., Karr, L., Ross, M., et al. (1994). Heterogeneity of Single Cell Cytokine Gene Expression in Clonal T Cell Populations. J.Exp.Med. 180, 1251-1262 
Carbo, A., Hontecillas, R., Kronsteiner, B., Viladomiu, M., Pedragosa, M., Lu, P., et al. (2013). Systems modeling of molecular mechanisms controlling cytokine-driven CD4+ T cell differentiation and phenotype plasticity. PLoS computational biology 9, e1003027.

doi:10.1371/journal.pcbi.1003027

Chang, J. T., Palanivel, V. R., Kinjyo, I., Schambach, F., Intlekofer, A. M., Banerjee, A., et al. (2007). Asymmetric T lymphocyte division in the initiation of adaptive immune responses. Science (New York, N.Y.) 315, 1687-1691. doi:10.1126/science.1139393

Chen, W., Jin, W., Hardegen, N., Lei, K.-j., Li, L., Marinos, N., et al. (2003). Conversion of Peripheral CD4+ CD25- Naive T Cells to CD4+ CD25+ Regulatory T Cells by TGF- $\beta$ Induction of Transcription Factor Foxp3. The Journal of Experimental Medicine 198, 1875-1886.

doi:10.1084/jem.20030152

Cortes, Y., Lotto, R. B., Malkin, D., Gerardo, J., Espinosa-Soto, C., Hartasa, D. A., et al. (2008). Floral morphogenesis: stochastic explorations of a gene network epigenetic landscape. PloS one 3, e3626. doi:10.1371/journal.pone.0003626

Cote-Sierra, J., Foucras, G., Guo, L., Chiodetti, L., Young, H. A., Hu-Li, J., et al. (2004). Interleukin 2 plays a central role in Th2 differentiation. Proceedings of the National Academy of Sciences of the United States of America 101, 3880-3885. doi:10.1073/pnas.0400339101

Crotty, S. (2014). T Follicular Helper Cell Differentiation, Function, and Roles in Disease. Immunity 41, 529-542. doi:10.1016/j.immuni.2014.10.004

Davidson, T. S., DiPaolo, R. J., Andersson, J., and Shevach, E. M. (2007). Cutting Edge: IL-2 Is Essential for TGFB Mediated Induction of Foxp3+ T Regulatory Cells. The Journal of Immunology 178, 4022-4026. doi:10.4049/jimmunol.178.7.4022

Davila-Velderrain, J., Villarreal, C., and Alvarez-Buylla, E. R. (2015). Reshaping the epigenetic landscape during early flower development: induction of attractor transitions by relative differences in gene decay rates. BMC systems biology 9, 20. doi:10.1186/s12918-015-0166-y

Davila-Velderraín, J. , Martínez-García, J. C., \& Álvarez-Buylla, E. R. (2017). Boolean Dynamic Modeling Approaches to Study Plant Gene Regulatory Networks: Integration, Validation, and Prediction. Humana Press, New York, NY. https://doi.org/10.1007/978-1-4939-7125-1_19

DuPage, M. and Bluestone, J. A. (2016). Harnessing the plasticity of CD4(+) T cells to treat immunemediated disease. Nat Rev Immunol 16, 149-163. doi:10.1038/nri.2015.18

Duque, G. A. and Descoteaux, A. (2014). Macrophage cytokines: Involvement in immunity and infectious diseases. Frontiers in Immunology 5, 491. doi:10.3389/fimmu.2014.00491

Eizenberg-Magar, I., Rimer, J., Zaretsky, I., Lara-Astiaso, D., Reich-Zeliger, S., and Friedman, N. (2017). Diverse continuum of CD4+ T-cell states is determined by hierarchical additive integration of cytokine signals. Proceedings of the National Academy of Sciences , 201615590doi:10.1073/pnas.1615590114

Fontenot JD Gavin MA, R. A. Y. (2003). Foxp3 programs the development and function of CD4+CD25+ regulatory T cells. Nat Immunol 4, 330-336 
Gagliani, N., Vesely, M. C. A., Iseppon, A., Brockmann, L., Xu, H., Palm, N. W., et al. (2015). Th17 cells transdifferentiate into regulatory $\mathrm{T}$ cells during resolution of inflammation. Nature 523, 221-5. doi:10.1038/nature14452

Grossman, Z. and Paul, W. E. (2015). Dynamic Tuning of Lymphocytes: Physiological Basis, Mechanisms, and Function*. Annual Review of Immunology 33, 677-713. doi:10.1146/ annurevimmunol-032712-100027

Haken, H. (1977). Synergetics (Berlin, Heidelberg: Springer Berlin Heidelberg). doi:10.1007/ 978-3642-96363-6

Hegazy, A. N., Peine, M., Helmstetter, C., Panse, I., Frohlich, A., Bergthaler, A., et al. (2010). Interferons " Direct Th2 Cell Reprogramming to Generate a Stable GATA-3+T-bet+ Cell Subset with Combined Th2 and Th1 Cell Functions. Immunity 32, 116-128. doi:10.1016/j.immuni.2009.12.004

Hori S Nomura T, S. S. (2003). Control of regulatory T cell development by the transcription factor Foxp3. Science 299, 1057-1061

Howes, A., Stimpson, P., Redford, P., Gabrysova, L., and O'Garra, A. (2014). Interleukin-10: Cytokines in Anti-inflammation and Tolerance. In Cytokine Frontiers: Regulation of Immune Responses in Health and Disease, vol. 6. 327-352. doi:10.1007/978-4-431-54442-5

Hsieh, C. S., Macatonia, S. E., Tripp, C. S., Wolf, S. F., O’Garra, A., and Murphy, K. M. (1993). Development of TH1 CD4+ T cells through IL-12 produced by Listeria-induced macrophages. Science (New York, N.Y.) 260, 547-9. doi:10.1126/science.8097338

Ivanov, I. I., McKenzie, B. S., Zhou, L., Tadokoro, C. E., Lepelley, A., Lafaille, J. J., et al. (2006). The Orphan Nuclear Receptor ROR $\gamma$ t Directs the Differentiation Program of Proinflammatory IL17+ T Helper Cells. Cell 126, 1121-1133. doi:10.1016/j.cell.2006.07.035

Johnston, R. J., Poholek, A. C., DiToro, D., Yusuf, I., Eto, D., Barnett, B., et al. (2009). Bcl6 and Blimp-1 Are Reciprocal and Antagonistic Regulators of T Follicular Helper Cell Differentiation. Science 325, 1006-1010. doi:10.1126/science. 1175870

Kaplan, M. H. (2013). Th9 cells: differentiation and disease. Immunological reviews 252, 104-15. doi:10.1111/imr.12028

Kauffman, S. (1969). Metabolic stability and epigenesis in randomly constructed genetic nets. Journal of Theoretical Biology 22, 437-467. doi:10.1016/0022-5193(69)90015-0

Kelso, A., Groves, P., Ramm, L., and Doyle, A. G. (1999). Single-cell analysis by RT-PCR reveals differential expression of multiple type 1 and 2 cytokine genes among cells within polarized CD4+ T cell populations. International immunology 11, 617-21. doi:10.1093/intimm/11.4.617

Kleinewietfeld, M. and Hafler, D. A. (2013). The plasticity of human Treg and Th17 cells and its role in autoimmunity. Seminars in immunology 25, 305-12. doi:10.1016/j.smim.2013.10.009

Koch, M. a., Tucker-Heard, G., Perdue, N. R., Killebrew, J. R., Urdahl, K. B., Campbell, D. J., et al. (2009). The transcription factor T-bet controls regulatory $\mathrm{T}$ cell homeostasis and function during type 1 inflammation. Nature immunology 10, 595-602. doi:10.1038/ni.1731 
Korn, T., Bettelli, E., Oukka, M., and Kuchroo, V. K. (2009). IL-17 and Th17 Cells. Annual Review of Immunology 27, 485-517. doi:10.1146/annurev.immunol.021908.132710

Le Gros, G., Ben-Sasson, S. Z., Seder, R., Finkelman, F. D., Paul, W. E., and Le Gros G Ben-Sasson SZ. (1990). Generation of interleukin 4 (IL-4)-producing cells in vivo and in vitro: IL-2 and IL-4 are required for in vitro generation of IL-4-producing cells. The Journal of experimental medicine 172, 921-929. doi:10.1017/CBO9781107415324.004

Lee, Y. K., Mukasa, R., Hatton, R. D., and Weaver, C. T. (2009a). Developmental plasticity of Th17 and Treg cells. Current Opinion in Immunology 21, 274-280. doi:10.1016/j.coi.2009.05.021

Lee, Y. K., Turner, H., Maynard, C. L., Oliver, J. R., Chen, D., Elson, C. O., et al. (2009b). Late Developmental Plasticity in the T Helper 17 Lineage. Immunity 30, 92-107.

doi:10.1016/j.immuni.2008. 11.005

Littman, D. R. and Rudensky, A. Y. (2010). Th17 and regulatory T cells in mediating and restraining inflammation. Cell 140, 845-858. doi:10.1016/j.cell.2010.02.021

Lu, Y., Hong, S., Li, H., Park, J., Hong, B., Wang, L., et al. (2012). Th9 cells promote antitumor immune responses in vivo. The Journal of clinical investigation 122, 4160-71. doi:10.1172/JCI65459 Magombedze, G., Reddy, P. B. J., Eda, S., and Ganusov, V. V. (2013). Cellular and population plasticity of helper CD4(+) T cell responses. Frontiers in physiology 4, 206. doi:10.3389/fphys.2013.00206

Martinez-Sanchez, M. E., Mendoza, L., Villarreal, C., and Alvarez-Buylla, E. R. (2015). A Minimal Regulatory Network of Extrinsic and Intrinsic Factors Recovers Observed Patterns of CD4+ T Cell Differentiation and Plasticity. PLoS computational biology 11, e1004324. doi:10.1371/journal.pcbi. 1004324

Martínez-Sosa, P. and Mendoza, L. (2013). The regulatory network that controls the differentiation of T lymphocytes. BioSystems 113, 96-103. doi:10.1016/j.biosystems.2013.05.007.

Mendoza, L. (2006). A network model for the control of the differentiation process in Th cells. BioSystems 84, 101-114. doi:10.1016/j.biosystems.2005.10.004

Mendoza, L., Thieffry, D., and Alvarez-Buylla, E. R. (1999). Genetic control of flower morphogenesis in Arabidopsis thaliana: a logical analysis. Bioinformatics (Oxford, England) 15, 593-606. doi:10.1093/ bioinformatics/15.7.593

Michalek, R. D., Gerriets, V. A., Jacobs, S. R., Macintyre, A. N., MacIver, N. J., Mason, E. F., et al. (2011). Cutting edge: distinct glycolytic and lipid oxidative metabolic programs are essential for effector and regulatory CD4+ T cell subsets. Journal of immunology (Baltimore, Md. : 1950) 186, 3299-303. doi:10.4049/jimmunol.1003613

Moore, K. W., de Waal Malefyt, R., Coffman, R. L., and O'Garra, A. (2001). Interleukin -10 and the I Nterleukin -10 R Eceptor. Annual Review of Immunology 19, 683-765.

doi:10.1146/annurev.immunol. 19.1.683

Murphy, E., Shibuya, K., Hosken, N., Openshaw, P., Maino, V., Davis, K., et al. (1996). Reversibility of T helper 1 and 2 populations is lost after long-term stimulation. The Journal of experimental medicine 183, 901-13. doi:10.1084/jem.183.3.901 
Murugaiyan, G., Mittal, A., Lopez-Diego, R., Maier, L. M., Anderson, D. E., and Weiner, H. L. (2009). IL-27 is a key regulator of IL-10 and IL-17 production by human CD4+ T cells. Journal of immunology (Baltimore, Md. : 1950) 183, 2435-2443. doi:10.4049/jimmunol.0900568

Naldi, A., Berenguier, D., Faure, A., Lopez, F., Thieffry, D., and Chaouiya, C. (2009). Logical modelling' of regulatory networks with GINsim 2.3. Bio Systems 97, 134-9. doi:10.1016/j.biosystems.2009.04.008

Naldi, A., Carneiro, J., Chaouiya, C., and Thieffry, D. (2010). Diversity and Plasticity of Th Cell Types Predicted from Regulatory Network Modelling. PLoS Computational Biology 6, e1000912. doi:10.1371/journal.pcbi.1000912

Naldi, A., Monteiro, P. T., Mussel, C., Kestler, H. A., Thieffry, D., Xenarios, I., et al. (2015). Cooperative " development of logical modelling standards and tools with CoLoMoTo. Bioinformatics 31, 1154-1159. doi:10.1093/bioinformatics/btv013

Noack, M. and Miossec, P. (2014). Th17 and regulatory T cell balance in autoimmune and inflammatory diseases. Autoimmunity Reviews 13, 668-677. doi:10.1016/j.autrev.2013.12.004

Novak, V., Perfiljeva I., and Mockor J., (1999) Mathematical Principles of Fuzzy Logic, Kluwer Academic Publishers, Boston.

Nurieva, R. I., Chung, Y., Martinez, G. J., Yang, X. O., Tanaka, S., Matskevitch, T. D., et al. (2009). Bc16 mediates the development of T follicular helper cells. Science (New York, N.Y.) 325, 1001-5. doi:10.1126/science. 1176676

Openshaw, P., Murphy, E. E., Hosken, N. A., Maino, V., Davis, K., Murphy, K., et al. (1995). Heterogeneity of intracellular cytokine synthesis at the single-cell level in polarized T helper 1 and $\mathrm{T}$ helper 2 populations. Journal of Experimental Medicine 182, 1357-1367.

doi:10.1084/jem.182.5.1357

Pandiyan, P., Zheng, L., Ishihara, S., Reed, J., and Lenardo, M. J. (2007). CD4+CD25+Foxp3+ regulatory $\mathrm{T}$ cells induce cytokine deprivation-mediated apoptosis of effector CD4+ T cells. Nature immunology 8, 1353-1362. doi:10.1038/ni1536

Panzer, M., Sitte, S., Wirth, S., Drexler, I., Sparwasser, T., and Voehringer, D. (2012). Rapid in vivo conversion of effector T cells into Th2 cells during helminth infection. Journal of Immunology 188, 615-23. doi:10.4049/jimmunol.1101164

Perez, V. L., Lederer, J. A., Lichtman, A. H., and Abbas, A. K. (1995). Stability of Th1 and Th2 populations. International Immunology 7, 869-875. doi:10.1093/intimm/7.5.869

Perez-Ruiz, R. V., Garc ' '1a-Ponce, B., Marsch-Mart'ınez, N., Ugartechea-Chirino, Y., VillajuanaBonequi, M., De Folter, S., et al. (2015). XAANTAL2 (AGL14) is an important component of the complex gene regulatory network that underlies arabidopsis shoot apical meristem transitions. Molecular Plant 8, 796-813. doi:10.1016/j.molp.2015.01.017

Pot, C., Jin, H., Awasthi, A., Liu, S. M., Lai, C.-Y., Madan, R., et al. (2009). Cutting edge: IL-27 induces the transcription factor c-Maf, cytokine IL-21, and the costimulatory receptor ICOS that 
coordinately act together to promote differentiation of IL-10-producing Tr1 cells. Journal of immunology (Baltimore, Md. : 1950) 183, 797-801. doi:10.4049/jimmunol.0901233

Roncarolo, M. G., Gregori, S., Battaglia, M., Bacchetta, R., Fleischhauer, K., and Levings, M. K. (2006). Interleukin-10-secreting type 1 regulatory T cells in rodents and humans. Immunological reviews 212, 28-50. doi:10.1111/j.0105-2896.2006.00420.x

Schmitt, E., Klein, M., and Bopp, T. (2014). Th9 cells, new players in adaptive immunity. Trends in immunology 35, 61-8. doi:10.1016/j.it.2013.10.004

Sozzani, S., Del Prete, A., and Bosisio, D. (2017). Dendritic cell recruitment and activation in autoimmunity. Journal of Autoimmunity doi:10.1016/j.jaut.2017.07.012

Stockinger, K. M. M. and Brigitta (2010). Effector T cell plasticity: flexibility in the face of changing circumstances 76, 211-220. doi:10.1007/s11103-011-9767-z.Plastid

Swain SL Weinberg AD, E. M. H. G. (1990). IL-4 directs the development of Th2-like helper effectors. J Immunol 145, 3796-3806

Szabo, S. J., Kim, S. T., Costa, G. L., Zhang, X., Fathman, C., and Glimcher, L. H. (2000). A Novel Transcription Factor, T-bet, Directs Th1 Lineage Commitment. Cell 100, 655-669. doi:10.1016/ S0092-8674(00)80702-3

Szabo, S. J., Sullivan, B. M., Peng, S. L., and Glimcher, L. H. (2003). Molecular mechanisms regulating Th1 immune responses. Annual review of immunology 21, 713-58.

doi:10.1146/annurev.immunol.21. 120601.140942

Travis, M. a. and Sheppard, D. (2014). TGF- $\beta$ activation and function in immunity. Annual review of immunology 32, 51-82. doi:10.1146/annurev-immunol-032713-120257

Veldhoen, M., Hocking, R. J., Atkins, C. J., Locksley, R. M., and Stockinger, B. (2006). TGF $\beta$ in the context of an inflammatory cytokine milieu supports de novo differentiation of IL-17-producing $\mathrm{T}$ cells. Immunity 24, 179-189. doi:10.1016/j.immuni.2006.01.001

Verbist, K. C., Guy, C. S., Milasta, S., Liedmann, S., Kaminski, M. M., Wang, R., et al. (2016). Metabolic ' maintenance of cell asymmetry following division in activated T lymphocytes. Nature 532, 389-93. doi:10.1038/nature17442

Villarreal, C., Padilla-Longoria, P., and Alvarez-Buylla, E. R. (2012). General Theory of Genotype to Phenotype Mapping : Derivation of Epigenetic Landscapes from N-Node Complex Gene Regulatory Networks. Physical Review Letters 118102, 1-5. doi:10.1103/PhysRevLett.109.118102

Wei, L., Laurence, A., and O'Shea, J. J. (2008). New insights into the roles of Stat5a/b and Stat3 in T cell development and differentiation. Seminars in cell \& developmental biology 19, 394-400.

doi:10.1016/j.semcdb.2008.07.011

Wohlfert, E. A., Grainger, J. R., Bouladoux, N., Konkel, J. E., Oldenhove, G., Ribeiro, C. H., et al. (2011). GATA3 controls Foxp3+ regulatory T cell fate during inflammation in mice. The Journal of clinical investigation 121, 4503-4515. doi:10.1172/JCI57456 
Xu, L., Kitani, A., Fuss, I., and Strober, W. (2007). Cutting Edge: Regulatory T Cells Induce CD4+CD25- Foxp3- T Cells or Are Self-Induced to Become Th17 Cells in the Absence of Exogenous TGF-. The Journal of Immunology 178, 6725-6729. doi:10.4049/jimmunol.178.11.6725

Yang, X. O., Nurieva, R., Martinez, G. J., Kang, H. S., Chung, Y., Pappu, B. P., et al. (2008). Molecular antagonism and plasticity of regulatory and inflammatory T cell programs. Immunity 29, 44-56. doi:10.1016/j.immuni.2008.05.007

Yu, D., Rao, S., Tsai, L. M., Lee, S. K., He, Y., Sutcliffe, E. L., et al. (2009). The transcriptional repressor Bcl-6 directs $\mathrm{T}$ follicular helper cell lineage commitment. Immunity 31, 457-468.

doi:10.1016/j.immuni. 2009.07.002

Zheng, Y., Josefowicz, S. Z., Kas, A., Chu, T.-T., Gavin, M. A., and Rudensky, A. Y. (2007). Genomewide analysis of Foxp3 target genes in developing and mature regulatory T cells. Nature 445, 93640. doi:10.1038/nature05563

Zheng W, F. R. A. (1997). The transcription factor GATA-3 is necessary and sufficient for Th2 cytokine gene expression in CD4 T cells. Cell 89, 587-596

Zhou, L., Ivanov, I. I., Spolski, R., Min, R., Shenderov, K., Egawa, T., et al. (2007). IL-6 programs T $\mathrm{H}-17$ cell differentiation by promoting sequential engagement of the IL-21 and IL-23 pathways. Nature immunology 8, 967-974. doi:10.1038/ni1488

Zhu, J., Yamane, H., and Paul, W. E. (2010). Differentiation of effector CD4 T cell populations (*). Annual review of immunology 28, 445-89. doi:10.1146/annurev-immunol-030409-101212 